\title{
In-plant 3D positioning system using point cloud data for remote decontamination machine
}

\author{
Yoshinori SATOH ${ }^{* * * *}$, Tetsuro AIKAWA***, Yuji KAWAGUCHI****, Naoya SAKAMOTO****, \\ Masahiro SAITO*,****, Masayuki KANEDA***** and Hitoshi SAKAI ${ }^{* * * * *}$ \\ *International Research Institute of Nuclear Decommissioning \\ 2-23-1, Shinbashi, Minato-ku, Tokyo 105-0003, Japan \\ **Power and Industrial Systems R\&D Center, Toshiba Corporation \\ 1, Komukaitoshiba-cho, Saiwai-ku, Kawasaki 212-8581, Japan \\ E-mail: yoshinori4.satoh@toshiba.co.jp \\ ***Isogo Nuclear Engineering Center, Toshiba Corporation \\ 8, Shinsugita-cho, Isogo-ku, Yokohama 235-8523, Japan \\ ${ }^{* * * *}$ Corporate Manufacturing Engineering Center, Toshiba Corporation \\ 33, Isogo-cho, Isogo-ku, Yokohama 235-0017, Japan
}

Received: 31 October 2016; Accepted: 31 May 2017

\begin{abstract}
Toshiba Corporation, a member of International Research Institute of Nuclear Decommissioning (IRID), has contributed to decontamination works throughout Fukushima Daiichi Nuclear Power Plant from outside ground to inside of the buildings. Speedy decontamination works allow workers to access and stay inside of the contaminated buildings for many hours, and as a result, all decommission works can be accelerated. Some remote decontamination machines to decontaminate the inside of the reactor buildings from a remote safe building have been developed for workers not to be radiated by high-level radiation. Conventionally, operators have remotely controlled the decontamination machine through multiple views of some remote surveillance cameras mounted on it. Because the position data such as GPS data is not available in the buildings, it was hard for operators to detect its absolute position and orientation in the building, and it took much time to recognize targets to be decontaminated. In order to reduce positioning time and make operation works easier, we constructed 3D positioning system to automatically detect the absolute 3D position of the decontamination machine in the reactor buildings from a remote safe building. Moreover, we can also keep records of decontamination works easily by tracking $3 \mathrm{D}$ position of the decontamination machine. This paper shows the overview of our approach of 3D positioning and a result of examinations in the mock-up facility that simulates a part of the inside of the reactor building at Fukushima Daiichi NPP.
\end{abstract}

Key words : Decontamination, Remote system, Image processing, 3D positioning, 3D point cloud

\section{Introduction}

Toshiba Corporation, a member of International Research Institute of Nuclear Decommissioning (IRID), has contributed to decontamination works throughout Fukushima Daiichi Nuclear Power Plant (NPP) from outside ground to inside of the building. Speedy decontamination work allows workers to access and stay the inside of the contaminated buildings for many hours, and as a result, all decommissioning works can be accelerated. Some remote decontamination machines to conduct decontamination from a remote safe building have been developed for workers not to be radiated by high-level radiation. Figure 1 shows the outside appearance of a decontamination machine mounted on the vehicle we developed (IRID, 2016). The upper part of this machine can be lifted up to the ceiling of the inside of buildings and can decontaminate the upper structures of the buildings with two controllable and expandable arms. It has multiple remote surveillance cameras to monitor surroundings of itself. Conventionally, operators have remotely controlled the machine through those multiple cameras. Because the accurate position data such as GPS data 
and geomagnetic data is not available in the building, it was hard for operators to detect its position and orientation in the building, and it takes much time to recognize targets to be decontaminated or obstructed objects. In order to reduce the positioning time and make the operation works easier, we have constructed 3D positioning system to automatically detect the absolute 3D position of the decontamination machine from a remote building (Aikawa, 2015) and improved this system.

The followings show the overview of the $3 \mathrm{D}$ positioning method and a result of the examination in a mock-up facility that simulates a part of the inside of the reactor building of Fukushima Daiichi NPP.

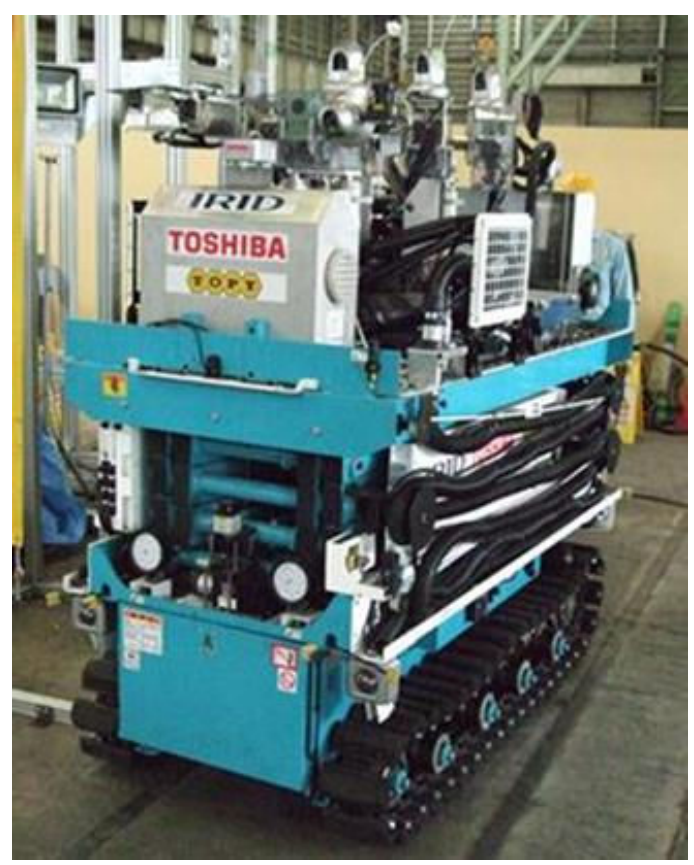

Fig. 1 Outside appearance of a decontamination machine mounted on the vehicle. The upper part of the machine can be lifted up to ceilings and decontaminate the surface of upper structures of the reactor buildings.

\section{Principle of 3D positioning}

Accurate decontamination needs accurate 3D positioning system to detect $3 \mathrm{D}$ position and orientation of the vehicle mounting the decontamination machine. In this case, because the position data such as GPS data and geomagnetic data is not available in the building, an alternative method to detect 3D position in the building is needed. Our 3D measurement method is based on the matching processing between 2D images obtained by cameras mounted on the decontamination machine and 3D point cloud data in the database we have collected and constructed beforehand.

Toshiba has been collecting the 3D point cloud data of the inside of the reactor buildings by using a 3D laser scanner after the disaster to survey the inside of the reactor buildings of Fukushima NPP (Kawaguchi, 2016). Figure 2 shows an example of 3D point cloud data obtained by a 3D laser scanner. Each gray-scale point in 3D point cloud data has 3D coordinate and the intensity of reflection at the point (Alshawabkeh and Naala, 2004) (Heritage and Large, 2009) (Kawaguchi, 2016). These 3D data are aligned according to absolute coordinate system based on the original drawings of the reactor buildings beforehand (Besl and McKay, 1992) (Kawaguchi et al., 2016). We can also check various sizes of space and target structures on the 3D point cloud data.

On the other hand, our decontamination machine has some remote surveillance cameras to monitor its surrounding environment remotely. Usually, operators remotely control the vehicle and the decontamination machine on the vehicle according to the multiple views through those cameras. From those surveillance cameras, we can get 2D images of the inside of the reactor building in real time. Our 3D positioning method is based on comparing these 2D images with the "known" 3D point cloud data.

Figure 3 shows the outline of the processing flow of our 3D measurement method. Figure 4 shows an example of 
the $2 \mathrm{D}$ images obtained by a camera mounted on the decontamination machine and an example of projection images of $3 \mathrm{D}$ point cloud data. The projection image shows the $2 \mathrm{D}$ plane image to be projected from $3 \mathrm{D}$ point cloud data. In this case, we usually use the views of the camera directing to the ceiling, because it is not easy for the views to be obstructed by other objects and the upper-side structures are not changed by other works in the near future.

In the processing flow, firstly, feature points of both 2D images obtained by the surveillance cameras and projection images of $3 \mathrm{D}$ point cloud data are extracted at the position which indicates characteristic region such as corners or crossing points by using the corner detection processing (Harris and Stephens, 1988) as shown in Fig. 4. These extracted feature points are compared between camera images and projection images by using the random sampling method (Fischler and Bolles, 1981) (Rousseeuw and Leroy, 2003), and the correspondences are detected as shown by lines in Fig. 5. The 3D position of 2D camera images, the 3D position of the decontamination machine, can be calculated based on the correspondence between these feature points.

Similar researches to compare 2D images with 3D data have been conducted (Meirerhold et al., 2010) (Becker and Haala, 2007). The difficulty of the matching processing in this case is to evaluate similarity between a feature point on camera images and one on projection images. Namely, evaluation value between $2 \mathrm{D}$ data and 3D data which has complex structures is needed according to each correspondence. Camera images are expressed by brightness and hue, and on the other hand, the projection images are expressed by intensity of laser light reflection. In order to set evaluation values to select optimal correspondence, our processing method adopts a matching method based on evaluation of gradient values of images without depending on absolute brightness or intensity.

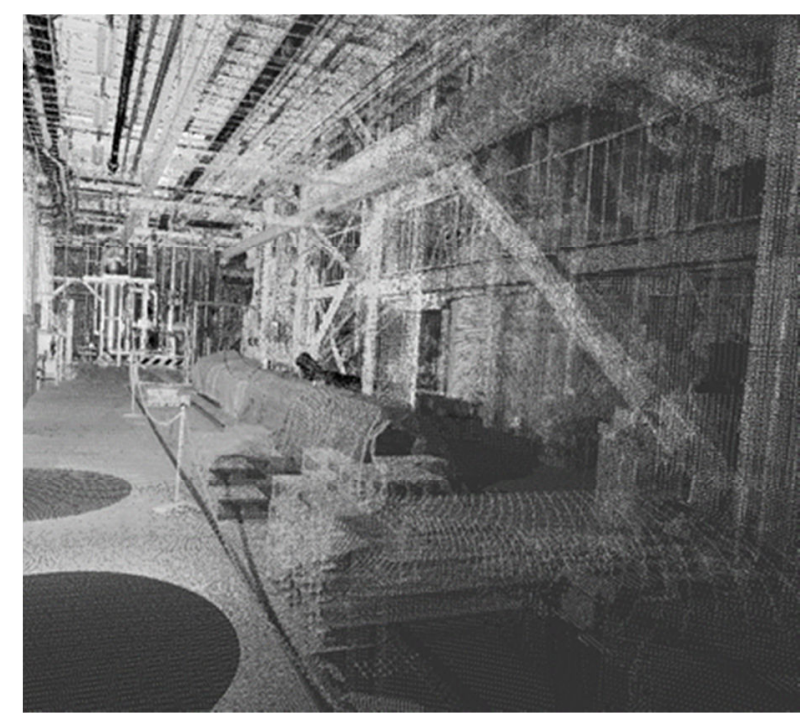

Fig. 2 An example of 3D point cloud data obtained by a 3D laser scanner.

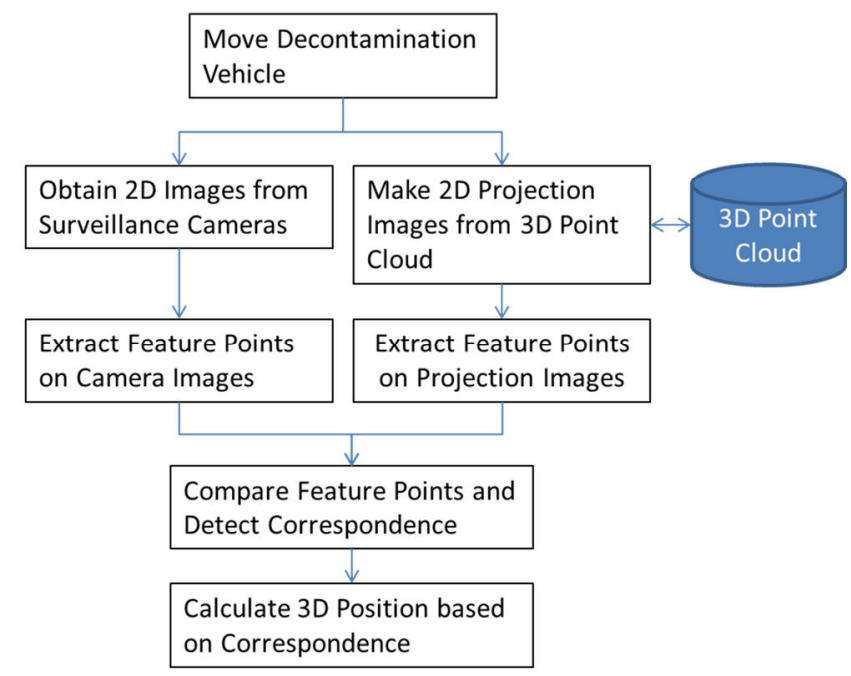

Fig. 3 Outline of the processing flow of 3D measurement method in-plant. 


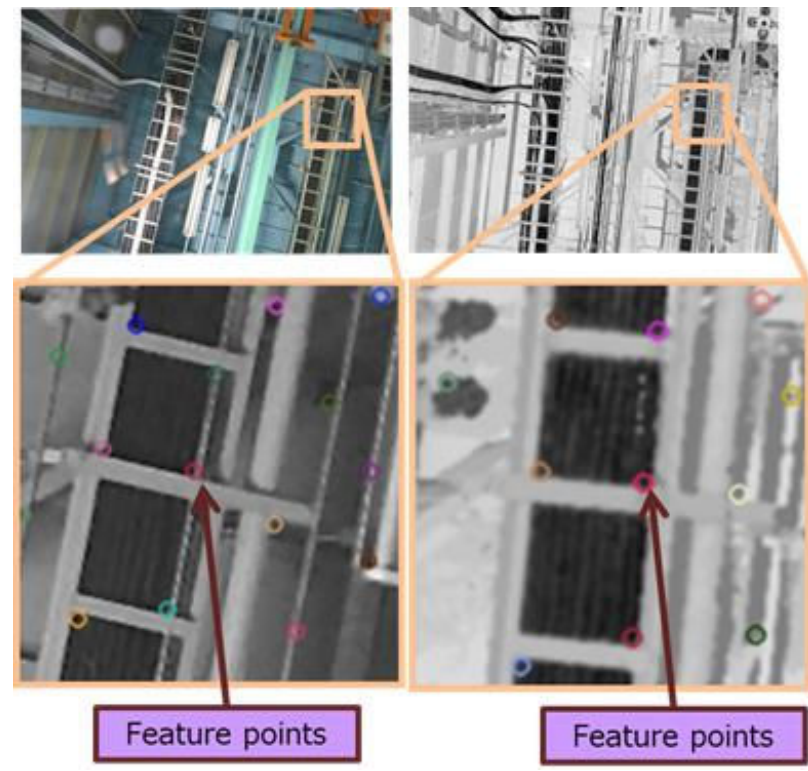

Fig. 4 Results of feature point extraction. The left image shows an image obtained by a mounted camera and the circles show feature points extracted according to corner detection. The right image shows an image projected from 3D data and the circles show feature points extracted according to corner detection.

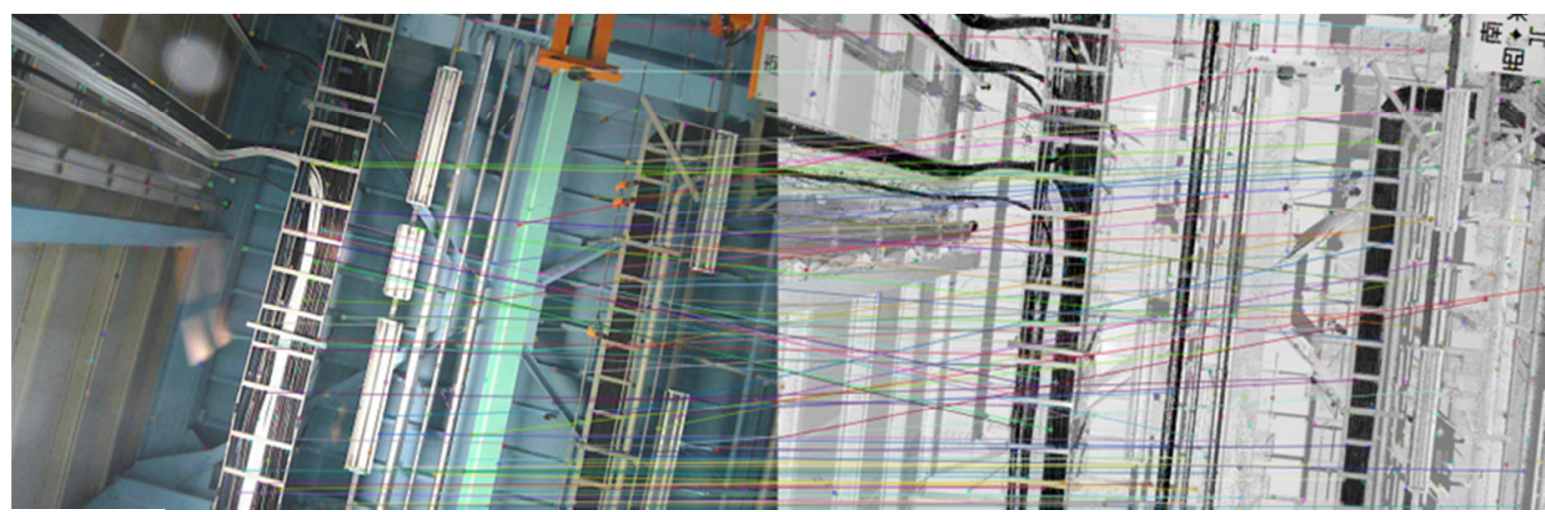

Fig. 5 Results of matching processing between feature points on a camera image and those of a projection image. The color lines show optimal correspondence between feature points.

\section{Examination result}

We conducted some examination to confirm the accuracy of our 3D measurement method indoors and to evaluate processing speed of it. This examination was conducted in the mock-up facility that simulates a part of the inside of the reactor building of Fukushima Daiichi NPP as shown in Fig. 6. The vehicle mounting the decontamination machine was remotely controlled by operators and ran in the mock-up facility for many times. Figure 7 shows an image obtained by a camera mounted on the decontamination machine and an image projected from the corresponded part of the 3D point cloud.

Figure 8 shows a screenshot of Graphical User Interface (GUI) of our software to show a result of 3D measurement. This GUI shows the 3D CG of the decontamination machine put in the 3D point cloud data of the mock-up facility according to the measurement result. The number on the left-upper side of the image shows the distance from the target position shown by the red rectangular CG. Figure 9 shows a look-down view on the X-Y plane corresponding to Fig. 8 . 
The difference between target position and the actual vehicle position detected by our 3D measurement method was confirmed and evaluated as shown in Fig. 10. We could detect 3D position of the decontamination machine from $30 \mathrm{~mm}$ to $80 \mathrm{~mm}$ difference. This accuracy will satisfy the control margin of the decontamination machine.

Measurement rate of this 3D measurement system was in 1 second. This rate will also be sufficient for operators to use at the actual NPP.

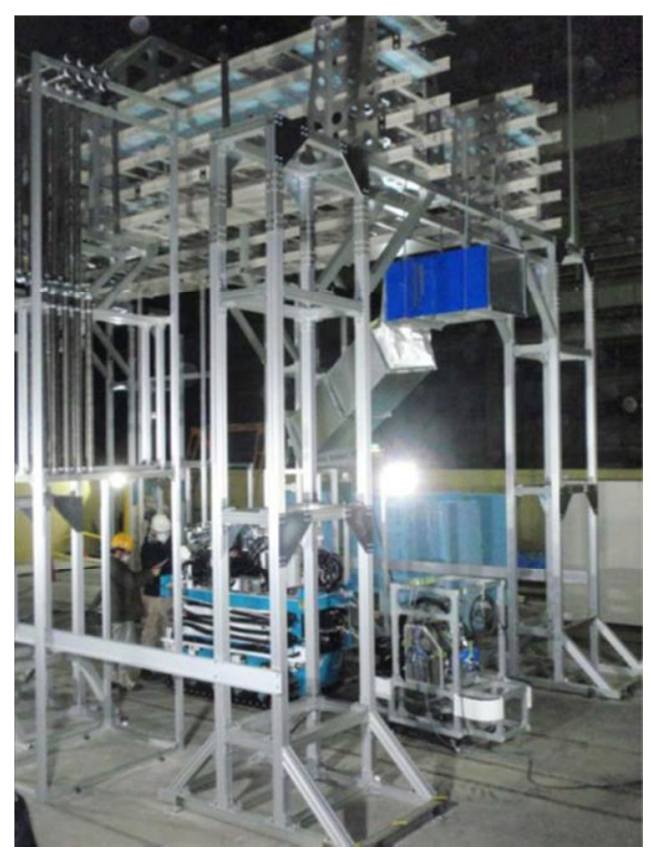

Fig. 6 Mock-up facility that simulates a part of inside of the reactor building of Fukushima Daiichi NPP and the decontamination machine running in the facility.
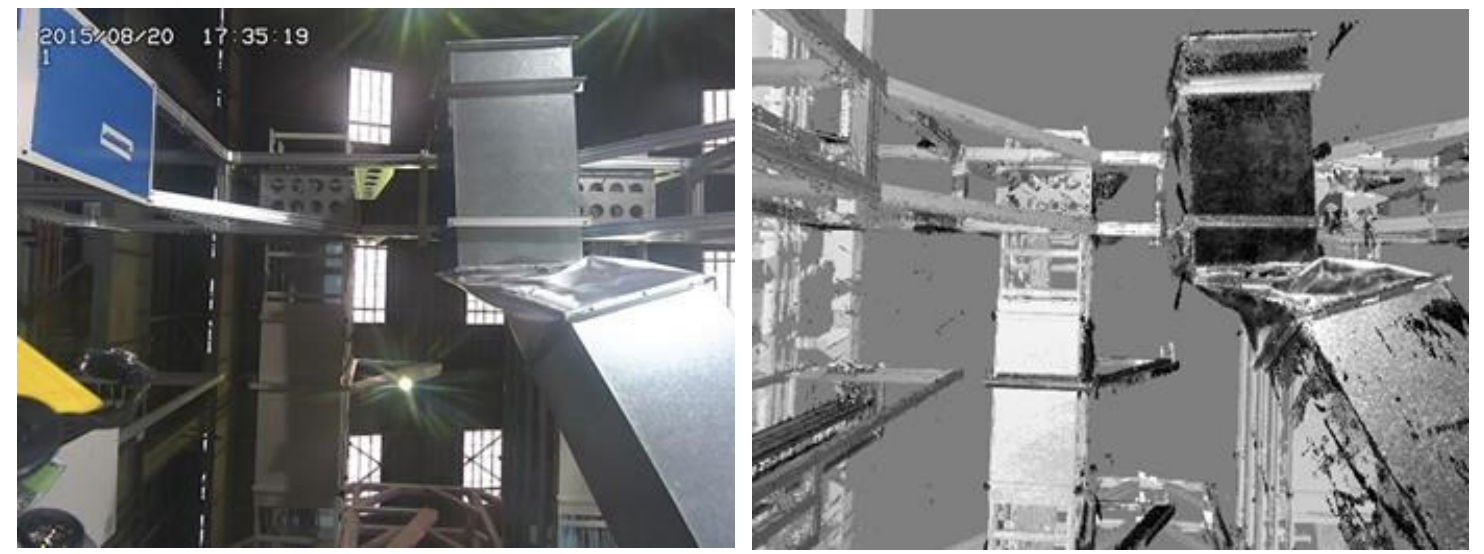

Fig. 7 An image obtained from surveillance camera mounted on vehicle (upper) and a projection image of 3D point cloud data at the counterpart (lower). 


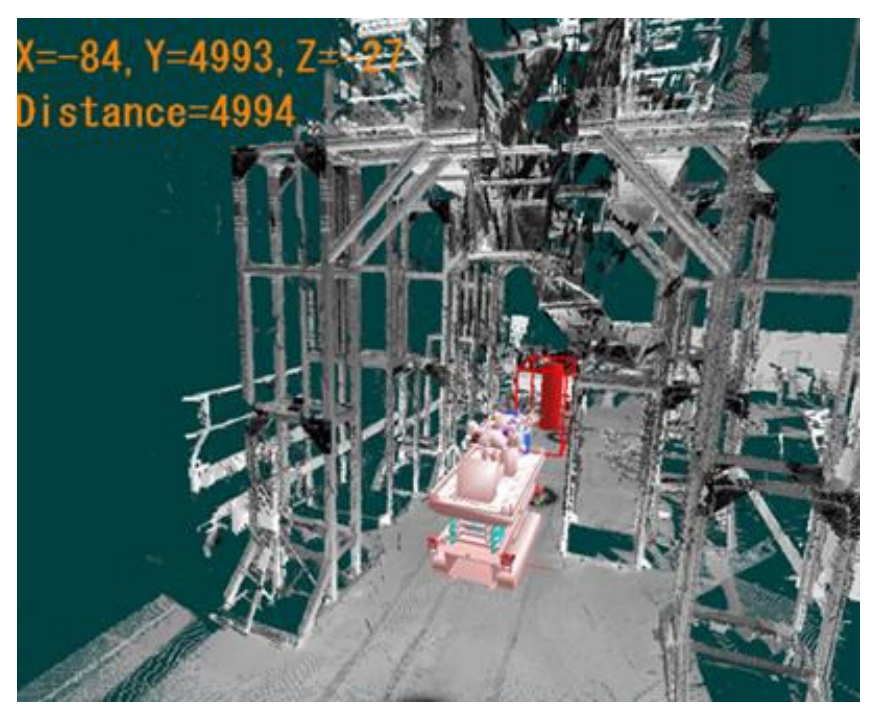

Fig. 8 Screenshot of UI to show the 3D CG of the decontamination machine in the 3D point cloud data of the mock-up facility according to a result of 3D measurement. The red rectangular shows target position for operators to stop the decontamination machine. The numbers on the left-upper side of the image show the difference between target position and the actual vehicle position.

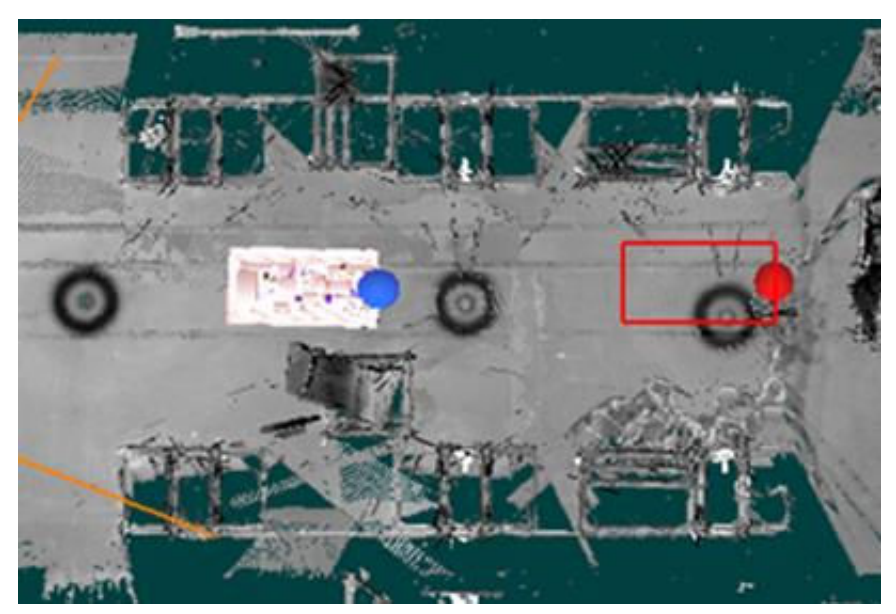

Fig. 9 Screenshot of a look-down view to show the position of the decontamination machine in the mock-up facility. The red rectangle shows target position for operators to stop the decontamination machine. 


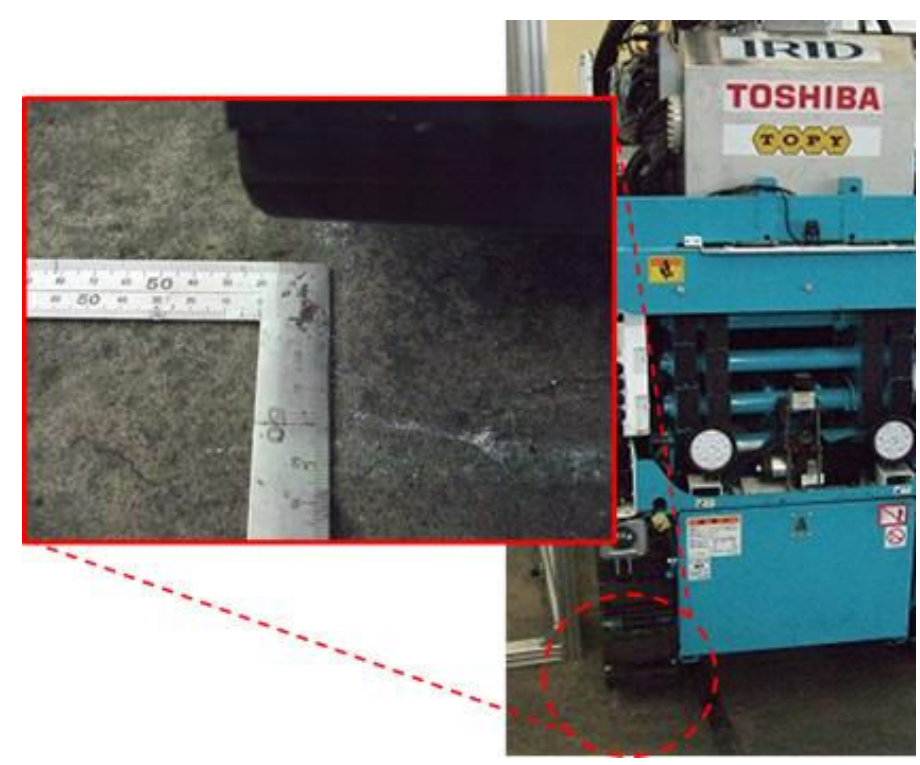

Fig. 10 Evaluation of accuracy of 3D positioning method. The difference between actual target position and actual vehicle position detected by our $3 \mathrm{D}$ positioning method is measured by a scale. The actual target position is marked on the ground with a white line.

\section{Summary}

We discussed a 3D positioning method based on 3D point cloud data to detect the 3D position of a decontamination machine in the reactor buildings, and showed experimental results to evaluate the accuracy of our method in the mock-up facility that simulates a part of the reactor building of Fukushima NPP. The accuracy and processing rate of the 3D measurement method satisfies the margin of the decontamination machine.

IRID and Toshiba are going to apply the decontamination machine including this 3D positioning system in actual decontamination works at Fukushima Daiichi NPP.

\section{Acknowledgement}

This work was supported by the grant-in-aid of Japanese Ministry of Economy, Trade and Industry.

\section{References}

Aikawa, T., Sakai, H., Sato, K., Hozumi, H., Murata, H. and Sakiyama, R., Development of Remote Decontamination Techniques for Reactor Building; Development of Decontamination Machine: Measurement of Decontamination Position using 3D data and Camera Image, Proceedings of 2015 Fall Meeting of the Automatic Energy Society of Japan, (2015), (in Japanese).

Alshawabkeh, Y. and Haala, N., Integration of digital photogrammetry and laser scanning for heritage documentation, XXth ISPRS Congress: Proceeding of Commission V (2009), pp.424-429.

Becker, S. and Haala, N., Combined feature extraction for facade reconstruction, the International Archives of the 
Photogrammetry, Remote Sensing and Spatial Information Sciences Workshop on Laser Scanning 2007 and CivLaser 2007 (2007), pp.44-49.

Besl, P.J., and McKay, N., A method for Registration of 3D Shapes, IEEE Transaction on Pattern Analysis and Machine Intelligence, Vol.14, No.2 (1992), pp.239-256.

Fischler, M.A., and Bolles, R.C., Random sample consensus: A paradigm for model fitting with applications to image analysis and automated cartography, Commun. ACM, No.24, Vol.6 (1981), pp.381-395.

Harris, C., and Stephens, M., A combined corner and edge detector, Proceedings of 4th Alvey Vision Conference (1988), pp.147-151.

Heritage, G. and Large, A., Laser Scanning for the Environmental Sciences (2009), John Wiley \& Sons, New York, USA.

International Research Institute for Nuclear Decommissioning (IRID), R\&D Topics: Development and utilization of dry ice blast decontamination equipment for high places (online), available from < http://irid.or.jp/en/topics/>, (accessed on 31 October, 2016).

Kawaguchi, Y., Satoh, Y., Matsumoto, S., Endoh, T. and Hatakeyama, M., Fast 3D Point Clouds Alignment and Efficient Laser Scanning Position Estimation using Dense Estimation, Proceedings of ANS 2016 International Congress on Advances in Nuclear Power Plants (2016).

Meirerhold, N., Spehr, M., Schilling, A., Gumhold, S. and Maas, H-G., Automatic feature matching between Digital Images and 2D representation of a 3D Laser scanner point cloud, the International Archives of the Photogrammetry, Remote Sensing and Spatial Information Sciences, Vol. XXXVIII, Part 5, Commission V Symposium (2010), pp.446-451.

Rousseeuw, P.J. and Leroy, A.M., Robust Regression and Outlier Detection (2003), John Wiley \& Sons, New York, USA. 